\title{
Illumination Threshold Study of Retinal Security by LED light
}

\author{
Shuyan Ren ${ }^{1, a}$, Liu Lu ${ }^{1, b}$, Li Zhao ${ }^{1, c}$, Yong Sun ${ }^{1, d}$ \\ ${ }^{1}$ D Keylaboratory of Information Sensing\&Intelligent Control, Tianjin University of Technology and \\ Education, Tianjin, 300300, China \\ 2a email: shuyan_ren@163.com, bemail:congratulate2008@163.com
}

Keywords: ERG; Retinal Security; Threshold; LED Light

\begin{abstract}
Medical experiments using white LED had been done to explain influence on visual function and Electroretinography (ERG) test results have been obtained under different illumination. From the experiments, different character of ERG was changed under different illumination. Compared with the control group, by the 300lux illumination, one of characters of ERG was showing the different trend. By the 1000lux illumination, some characters of ERG was showing distinguished difference. So 300lux illumination can be determined as the safety threshold wavelength of white LED light source initially. Experimental Study of the Retinal Security by LED light will provide theoretical basis to indoors application of LED light.
\end{abstract}

\section{Introduction}

Retinal light damage phenomenon is conducted by visual pigment photoreceptor cell damage and death, which depends on the light wavelength, light energy, duration, irradiation methods and so on[1]. Long-term light can cause a series of defects of transmission mechanism, cell death and retinal degeneration.

Studies have shown that prolonged exposure to bright light can cause adverse effects on retinal func-tion. Many scholars used a variety of experimental animals to demonstrate the effects of light on the re-tina and its mechanism [2,3].The causes of light im-pact on the retina are deduced to three aspects, which are thermal damage, mechanical damage and photochemical damage [4-6]. Thermal damage is produced from high energy absorbed by the tissue, which makes local tissue temperature increase. When the tissue temperature exceeds a certain limit, all kinds of protein components within the organization produce solidification and conduct damage. Me-chanical damage is that tissue receives strong light (such as laser) in a very short time, and organization instantaneous changes by the photon changing in an instant to mechanical damage tissue. Photochemical damage is pathological changes in retinal tissue which is caused by not obvious temperature rising, low energy and relatively long time illumination.

LED semiconductor light-emitting device as the fourth generation of light source, will soon be widely used in indoors, which can realize lighting and communication functions at the same time. Explor-ing healthy and comfortable designing methods of indoor LED lighting, or providing theoretical basis and technical support is important to improve the general health level of human being and has enorm-ous social benefits. In this paper,

White LED is used as the light source to do medical research to find the LED light source effect on the visual function. Under the same conditions of irra-diating time, the retina damages have been compared by different illumination of white LED on healthy rats to get safer white LED illumination range, which will provide a theoretical reference for further applications of LED indoors.

\section{The Interaction Principle of Light and Biological Tissue}

Rod cells and cone cells are two types of visual cells in the human eye retina, and these cells have a photosensitive substance called sensitive pigment. Based on mechanism of interaction between light and biological tissues[7],the visual cells generate pulses by the light. These pulses are 
posted to the brain's visual centers by the optic nerve. When photosensitive pigments of the visual cells were stimulated by visible light, photons of a certain wavelength would be absorbed. The electronics within the biological molecules jumped to a certain level and caused the variation of the biological molecules. When these effects are not strong enough, damage does not emerge, and some beneficial effects of biological stimuli have been produced. However, when the stimulus is too strong, it will cause irreversible damage to the visual [8-9].

In order to assess retinal damage degree in rats by the white LED, relative animal experiments have been done. Selecting 40 SD rats of 8-10 weeks old, and divide them into 4 groups randomly. One group is named control group, the others are experimental groups. Every group whith10 rats were irradiated by different illumination(200lux, 300lux and 1000lux) and intermittent light(12 hours on and 12hours off) for 7 days. After 24 hours dark adaptation, ERG was got to access retinal function.

\section{White LED}

According to the experimental requirements, cages of rats and light sources are designed. Selecting white LED diodes as light source, the light-emitting surface is $400 \mathrm{~mm} * 600 \mathrm{~mm}$. Adding a piece of glass as a scatter to enhance the uniformity of illumination, the method of segmentation controlling is used to ensure the stability of photoluminescence. The spectral of light is shown as Fig1. Under $1000 l u x$, peak wavelength is about $442.1 \mathrm{~nm}$, spectral half width is $23.6 \mathrm{~nm}$, the light flux is $1049 \mathrm{~lm}$ and the radiant flux is $3.482 \mathrm{w}$.

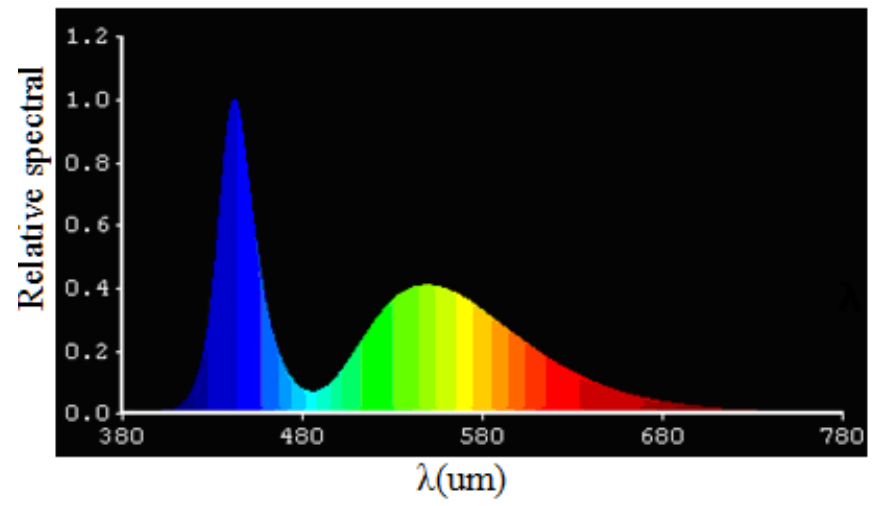

Fig1 Test spectral of LED light source

\section{Experimental data analysis}

After the human eye's retina is stimulated by light, photochemical and photoelectric reactions will occur in the visual receptors, which produce potential changes and form nerve impulses passing to bipolar cells, ganglion cells, optic nerve, optic chiasm, optic tract, lateral geniculate body. Optic radiation terminates in the cerebral cortex of the calcarine visual cortex. After the retina is stimulated by the full-field (Ganzfeld) flash, the electrical responses sum of the retina neurons and non-neuronal cells are recorded by corneal. It represents the electrical activity of the retinal cells of every layer. Diagram of electroretinogram (ERG) [10,11] is shown in figure 2.

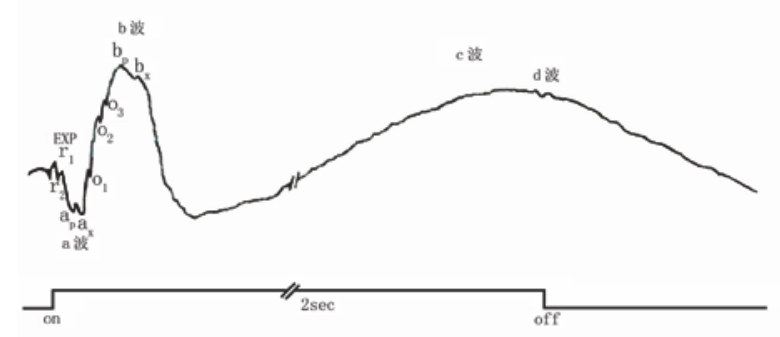

Fig2 Schematic diagram of ERG component

The common clinical abnormal ERG is mainly embodied in five different types. The amplitude 
of B wave of hypertypic ERG exceeds the normal average value over 30\%. a, b wave amplitude of low ERG decreases, and the range should be lower than the normal average value over $30 \%$. For the negative wave of ERG , a wave is deep and wide, and b wave is small or disappeared. For delayed-type ERG's, a and b wave amplitude is normal, but the peak time will delay by standard flash recording. The composition of flat type ERG will be submerged in the baseline noise.

In the visual electrophysiological experiments, RolanConsult's eye electrophysiological systems and Retisystem2.26 software are used to measure ERG. Corneal electrode, and the reference electrode and the ground electrode are made of stainless steel needle.

Compared with the rat retinal electrophysiological data stimulated by different illumination, the light and electron microscopy results, four typical characters have been taken as parameter vectors, which are incubation period of a wave, amplitude of a wave, incubation period of $b$ wave and amplitude of b wave. For the study of multidimensional variables, PCA method was adopted to reduce the dimensions, then do the cluster analysis of retinal electrophysiological data. Compared with control group, there are no differences in the retinal electrophysiological data by 200lux illumination. there are some kind of difference trend in the retinal electrophysiological data by 300lux illumination, because the data are more likely divided. there are distinguished differences in the retinal electrophysiological data by 1000lux illumination.

According to the results of above medical experiments, ANOVA model (variation of main characters of ERG) was studied under different illumination, which were shown in Fig3 and Fig4. Compared with the control group, by the 300lux illumination, one of characters of ERG was showing the different trend. By the 1000lux illumination, some characters of ERG was showing distinguished difference.

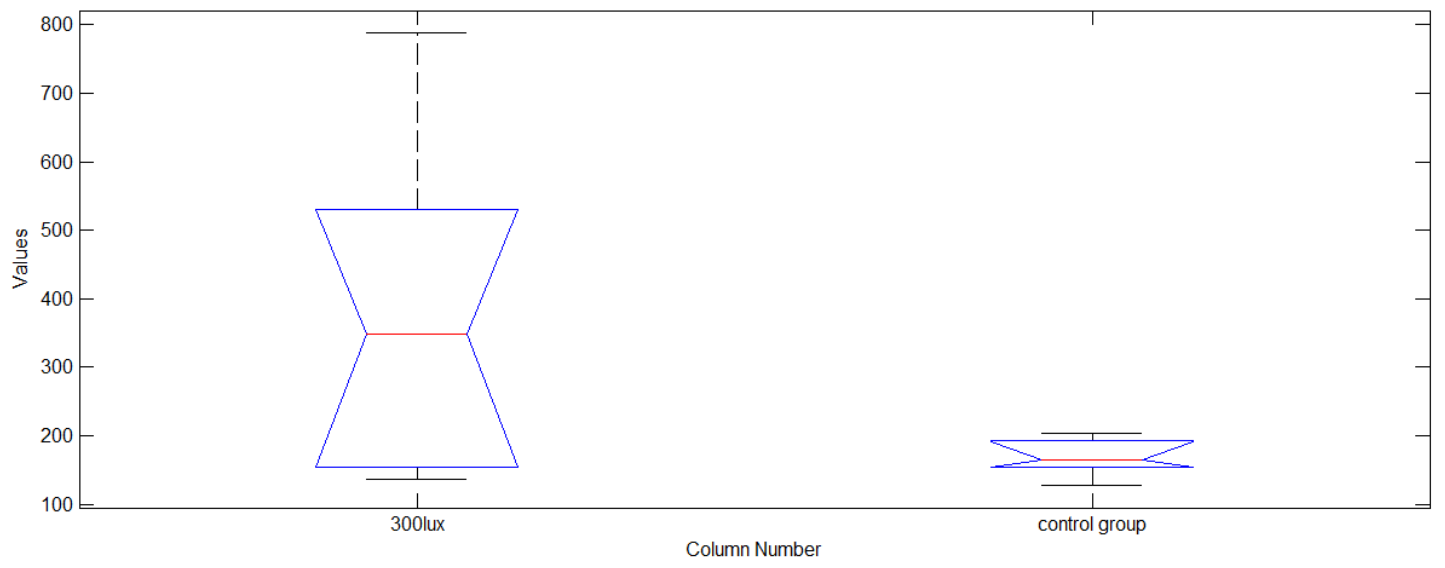

Fig3 ANOVA by 300lux

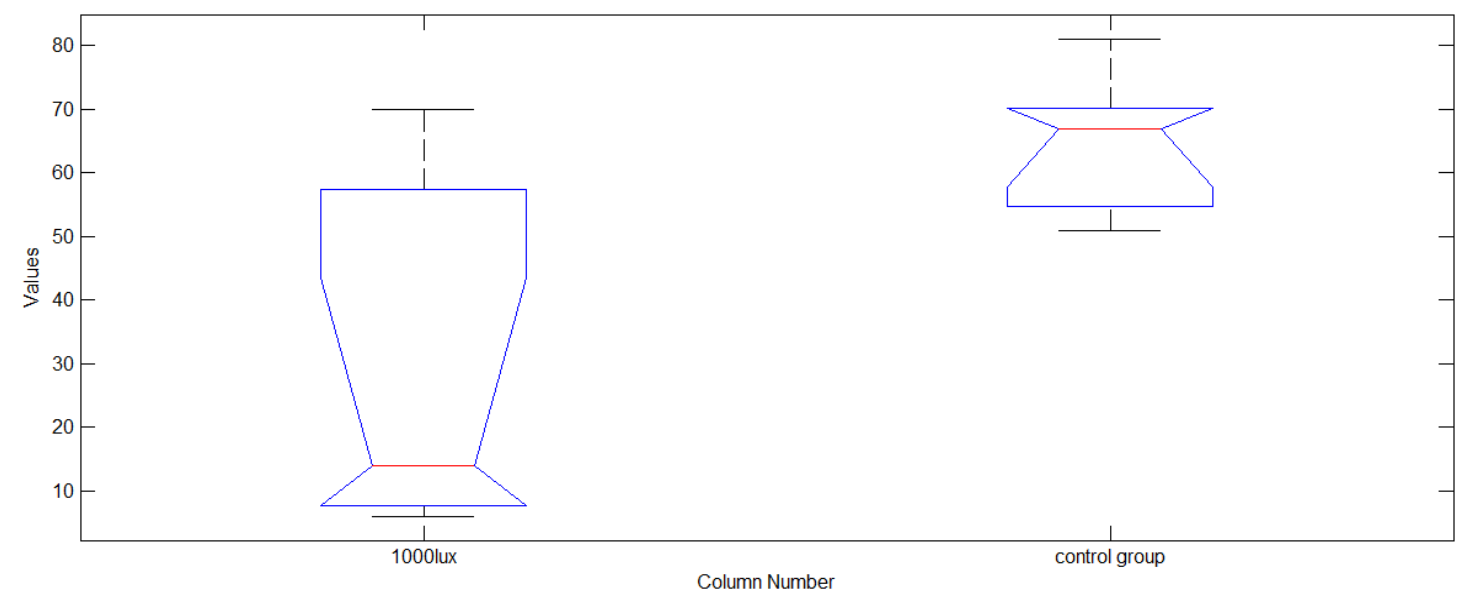

Fig4 ANOVA by 1000lux

According to results, it is safe for 300lux illumination of white LED indoors. But 1000lux illumination of white LED can cause retinal damage. 


\section{Conclusion}

In this paper, basic medical experiments were done to study the variation of main characters in rat ERG under different illumination. From the Figs, compared with that of control group, the main characteristics of rat ERG have no significant statistical differences under 300lux illumination of white LED. But there are obvious statistical differences under 1000lux illumination of white LED. So the extent of damage of retinal is related to the illumination of white LED. From the experiments, 300lux illumination was regarded as the referenced safety threshold and the extent of damage is related to the illumination of white LED.

\section{Acknowledgement}

The National Natural Science Fund Project "LED light source of the retina medical security and visual function influence mechanism research"(No.61178081) and The Research and Development fund(No. KJY11-09), Natural Science Foundation of Tianjin (14JCZJC36300).

\section{References}

[1] Shang YM, Wang GS, Sliney D, et al.White Light-Emitting Diodes (LEDs) at Domestic Lighting Levels and Retinal Injury in a Rat Model[J].Environ Health Perspect, 2014, 122(3):269-276

[2] Desmet, K.D, et al. 2006, Clinical and experimental applications of NIR-LED photobiomodulation[J]. Photomed Laser Surg, 24(2): 121-128.

[3] Suzdalienko A., Galkins I. 2010, Research and Development of LED Driver with Power Line Communication for Intelligent Lighting[J], Proceedings of NEXT 2010, Finland, Turku, 78-85.

[4] Komine Toshihiko, Haruyama Shiniehiro, Nakagawa Masao. 2005, A study of shadowing on indoor Visible-light wireless communication utilizing Plural white LED lightings[J]. Wireless Personal communications, 34(1-2):211-22.

[5] PennJS, Anderson RE. 1991, Effect of light history on the rat retina[J]. Prog Ret Res, 11:75.

[6] Young RW. 1988, Solar radiation and age-related macular degeneration[J]. Sury Ophihalmol, 32:252.

[7] Ash, C, G. Town, and P. Bjerring. 2008, Relevance of the structure of time-resolved spectral output to light-tissue interaction using intense pulsed light[J]. Lasers Surg Med, 40(2): 83-92.

[8] Dall Agnol, et al. 2009, Comparative analysis of coherent light action (laser) versus non-coherent light (light-emitting diode) for tissue repair in diabetic rats [J]. Lasers Med Sci, 24(6): 909-916.

[9] Leal Junior, E.C., et al., 2009, Effect of cluster multi-diode light emitting diode therapy (LEDT) on exercise-induced skeletal muscle fatigue and skeletal muscle recovery in humans. Lasers Surg Med, 41(8): 572-577.

[10] Li Qiaolian, Tang Renhong and Yi Junhui. 2013, Application ERG assess changes in retinal function with high myopia,Chinese and foreign medical research, 11(12):151-153.

[11] Wang Hui Wu Xingwei Zhu Jianfeng Chen Fenge Wu Ying Sun Yong Gong Yuanyuan. 2008, Determination of $[\mathrm{J}]$. high myopia macular retinal thickness and multifocal ERG.Journal of Optometry. 10 (05) :332-334. 